\title{
Accompagner la professionnalisation des formateurs en simulation médicale
}

Accompany the professionalization of the trainers in medical simulation

Bruno Bastiani, Bernard Calmettes, Vincent Minville et Fouad Marhar

\section{OpenEdition}

\section{Journals}

\section{Édition électronique}

URL : https://journals.openedition.org/educationdidactique/2659

DOI : 10.4000/educationdidactique.2659

ISSN : 2111-4838

\section{Éditeur}

Presses universitaires de Rennes

\section{Édition imprimée}

Date de publication : 20 juin 2017

Pagination : 63-79

ISBN : 978-2-7535-6460-2

ISSN : 1956-3485

Référence électronique

Bruno Bastiani, Bernard Calmettes, Vincent Minville et Fouad Marhar, « Accompagner la professionnalisation des formateurs en simulation médicale », Éducation et didactique [En ligne], 11-1 | 2017, mis en ligne le 20 juin 2019, consulté le 16 août 2022. URL : http://journals.openedition.org/ educationdidactique/2659; DOI : https://doi.org/10.4000/educationdidactique.2659 


\title{
ACCOMPAGNER LA PROFESSIONNALISATION DES FORMATEURS EN SIMULATION MÉDICALE
}

\author{
Bruno Bastiani, \\ doctorant en sciences de l'éducation, université Toulouse Jean Jaurès \\ Bernard Calmettes, \\ maître de conférences-HDR en sciences de l'éducation, université Toulouse Jean Jaurès \\ Vincent Minville, \\ Fouad Marhar, \\ chef de clinique universitaire assistant des hôpitaux de Toulouse, université Toulouse Paul Sabatier
} professeur des universités et praticien hospitalier en anesthésie-réanimation, université Toulouse Paul Sabatier

Notre recherche prend comme objet d'étude les situations de débriefing faisant suite à des séances de formation, destinées à des équipes d'anesthésie-réanimation (médecins anesthésistes-réanimateurs et infirmiers anesthésistes), mettant en œuvre des dispositifs de simulation comme réplique de bloc opératoire. L'objectif sera, ensuite, d'élaborer une formation de formateurs structurée à partir de ressources médicales et de propositions issues des sciences humaines. Dans cet article, nous présentons une partie de ce travail de recherche. Nous nous intéressons plus particulièrement à la dynamique des activités des formateurs et des formés en relation avec le développement des compétences non-techniques de ces derniers, pendant les débriefings. Nos résultats nous conduisent à faire des propositions pour la formation.

Mots-clés : formation, médecin anesthésiste-réanimateur, simulation, compétence non-technique, débriefing.

Accompany the professionalization of the trainers in medical simulation

Our research focuses on debriefing situations following training sessions for anesthesiologists using simulation devices to replicate an operating room. Our long term objective is to create a training-of-trainers module based on medical resources and propositions from educational sciences. Part of this research is presented in this article. We focus more particularly on the interactions between trainers and trainees that occur during debriefings, in relation with the development of nontechnical skills in trainees. Our results lead us to propose changes in training practices.

Keywords: training sessions, anesthesiologist, simulation, non-technical skills, debriefing. 


\section{INTRODUCTION}

La simulation en anesthésie-réanimation : pour quelles finalités?

Le recours à la simulation, dans le champ de la santé et plus particulièrement en anesthésie-réanimation, correspond à une pratique déjà ancienne. Le professeur Peter Safar (1958) collabore, dans les années soixante, avec l'entreprise norvégienne Laerdal (fabricant de jouets) au développement d'un mannequin permettant l'entraînement à certains gestes médicaux techniques: actes médicaux sur le patient ou préparation individuelle de ces actes. Le développement de l'informatique et son intégration dans des outils de simulation amènent chercheurs, formateurs et médecins à des modifications de pratiques importantes, en portant notamment une attention accrue au facteur humain dans la gestion des crises en anesthésie (cf. par exemple le Comprehensive Anesthesia Simulation Environment, CASE, développé par Gaba et son équipe, 1988).

Schaefer, Helmreich et Schneidegger. (1994) constatent ainsi que les attitudes des professionnels de santé, le type d'organisation et les paramètres culturels de travail ont une influence importante sur la communication, la distribution des activités et la résolution des conflits. Ces différents travaux et le rapport « to Err is Human » (1999) conduisent à une évolution des situations de formations à partir d'une utilisation accrue de la simulation, utilisée notamment pour sa dimension éthique.

Les chercheurs et les praticiens orientent alors leurs réflexions au-delà des aspects strictement techniques, longtemps perçus comme unique axe de formation, vers le développement de compétences non-techniques visant l'activité collective dans les interventions en bloc opératoire (Weinger \& Slagle, 2001 ; Fletcher, McGeorge, Flin, Glavin \& Maran, 2002 ; Cooper, Newbower, Long \& McPeek, 2002 ; Phipps, Meakin, Beatty, Nsoedo \& Parker, 2008).

La simulation en anesthésie-réanimation : description générale des dispositifs de formation étudiés

Les simulations sont réalisées dans des répliques de blocs opératoires, avec un patient matériellement représenté par un mannequin reproduisant l'anatomie d'un patient (Fig. 1).

Figure 1.

Configuration des lieux

\section{Configuration des lieux}

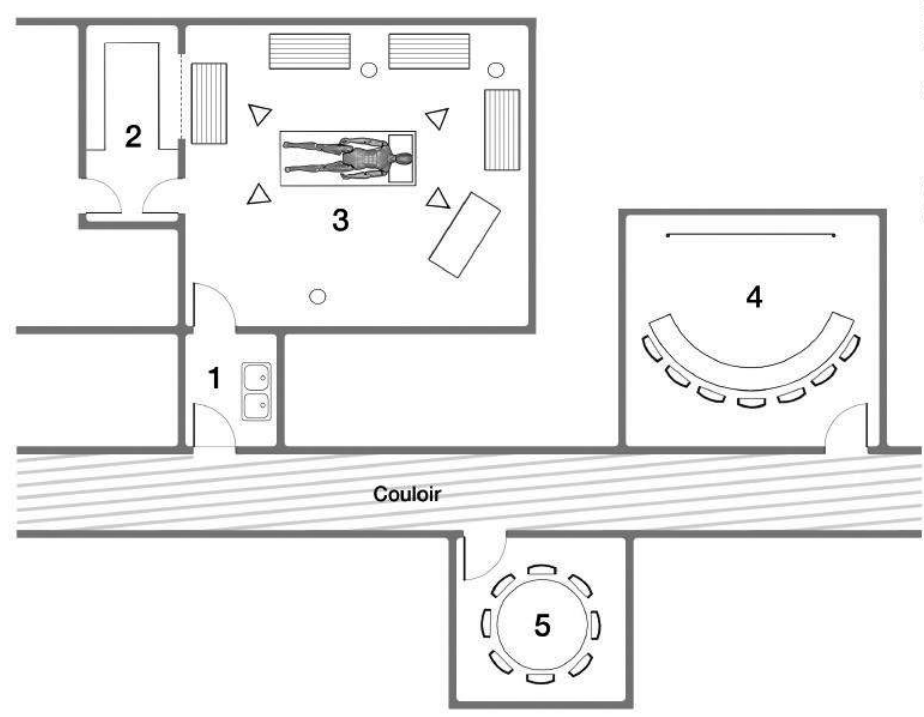

1. Sas d'entrée du bloc opératoire

2. Salle de contrôle

3. Salle de stimulation avec mannequin :
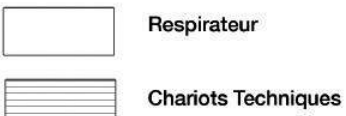

Caméras

$\triangle$ Micros

4. Salle des observateurs

5. Salle de repos 


\section{Salle de simulation avec mannequin (bloc) :}

Cette salle est une réplique de bloc opératoire avec tout le matériel placé habituellement dans un bloc : table d'opération, éclairage opératoire (scialytique), matériel d'anesthésie, moniteurs de surveillance des paramètres vitaux du patient, chariot de réanimation avec tous les médicaments (curare, adrénaline, produits anesthésiques...) et les instruments (seringues, sondes, défibrillateur, ballon et masque facial...). Elle est équipée de trois caméras et de quatre micros afin d'enregistrer l'ensemble des actes et des interactions des formés MAR et IADE.

\section{Les formés présents dans le bloc:}

Dans un scénario standard, la simulation en anesthésie dans le bloc met en scène le binôme traditionnel retrouvé dans les blocs opératoires : un Médecin Anesthésiste-Réanimateur (MAR) et un Infirmier Anesthésiste Diplômé d'État (IADE). Le MAR est un interne en médecine (pouvant participer à la simulation à partir du $3^{\grave{e}}$ semestre de sa spécialisation d'anesthésie-réanimation) alors que l'IADE est un personnel paramédical diplômé, il vient dans le cadre d'un Développement Professionnel Continu (DPC, dispositif de formation obligatoire une fois par an pour les paramédicaux).

\section{Salle de contrôle :}

La salle est équipée de plusieurs écrans de contrôle et du dispositif de commande du mannequin. Un formateur suit le déroulement de la simulation par l'intermédiaire de ces écrans de contrôle, il a pour mission d'adapter les paramètres du mannequin par rapport aux actions menées par les formés durant la simulation.

\section{Salle des observateurs :}

Dans cette salle se trouvent le reste du groupe des formés (MAR et IADE) de la journée de formation et un formateur. Ils observent le déroulement de la séance de simulation sur un écran géant qui permet de visualiser la situation et les constantes du mannequin. Ils participent ensuite au débriefing.
Certains d'entre eux peuvent être appelés à participer à la séance de simulation si les participants du bloc demandent de l'aide. C'est dans cette salle que se déroule également le débriefing.

\section{Salle de repos :}

Lorsque le scénario est construit de telle sorte que l'appel à l'aide est une nécessité logique, certains formés, qui seront ceux appelés en renfort, attendent cet appel dans une salle de repos qui ne dispose d'aucun système de visualisation du bloc. Ainsi, ils ne bénéficient pas d'informations préalables à leur entrée concernant le déroulement de la séance. Cette démarche a pour but de préserver le caractère authentique d'une situation réelle dans laquelle le renfort n'a pas connaissance a priori des évènements avant d'arriver dans le bloc.

\section{Animation de la formation :}

La formation est animée au minimum par une équipe de supervision constituée d'un médecin anesthésiste-réanimateur (MAR formateur) et de deux infirmiers anesthésistes (IADE formateur) expérimentés, qui bénéficient d'une reconnaissance croisée de leurs pairs sur le plan technique.

De façon standard, nous pourrions distribuer les rôles des formateurs tels que :

- l'un observe la situation par l'intermédiaire d'écrans dans un poste de contrôle, il réadapte et dirige les paramètres du mannequin en fonction de l'action des formés ;

- un autre reste avec les observateurs et visionne le cas par écran interposé ;

- le troisième peut intervenir dans le bloc opératoire pour jouer le rôle d'un professionnel de santé soit comme un élément facilitateur soit comme un élément perturbateur ; il peut, par exemple, jouer le rôle d'un chirurgien contraint d'opérer dans des durées strictes alors que l'induction anesthésique (administration et injection de produits anesthésiants en plusieurs phases) prend plus de temps que prévu. Ponctuellement, d'autres formateurs peuvent intervenir en fonction des scénarios afin de jouer le rôle du chirurgien, de l'Infirmier de Bloc Opératoire (IBODE), de l'AideSoignant (AS), d'un manipulateur radio, etc. 


\section{Le scénario :}

Les simulations sont interactives dites «pleine échelle ». Par exemple, par le pilotage du mannequin ou sa programmation en amont, l'abord des voies aériennes peut être limité, ce qui peut rendre impossible la mise en œuvre d'une intubation, acte qui consiste à introduire un tube dans la trachée pour contrôler les voies aériennes pendant l'anesthésie. Les mouvements respiratoires spontanés ainsi que les bruits auscultatoires peuvent être modifiés; le mannequin peut parler ou pleurer ; ses paramètres physiologiques sont modifiables en fonction de l'intervention.

Chaque scénario ${ }^{1}$ permet de simuler une situation critique pour un patient. Il s'agit le plus souvent d'un cas clinique rare nécessitant la mise en œuvre de compétences variées. Ce cas doit être résolu rapidement pour éviter l'engagement du pronostic vital chez le patient. Celui-ci peut, par exemple, avoir une réaction allergique au type d'anesthésique administré. Les paramètres physiologiques du mannequin sont alors effectivement modifiés entraînant un arrêt respiratoire et cardiaque. L'équipe en présence dans le bloc a la possibilité de faire appel à un renfort venant de la salle des observateurs ou de la salle de repos.

\section{Les étapes d'une séance de simulation:}

- le briefing permet de préciser le déroulement de la simulation, de décrire l'organisation du bloc opératoire et les fonctions du mannequin, de mettre en confiance les étudiants, de faire un essai de laryngoscope, instrument utilisé pour l'intubation du patient ; - la situation simulée commence par l'examen de la fiche «patient» (caractéristiques du patient, type d'intervention...) et se poursuit par la mise en œuvre d'un scénario prédéterminé par les formateurs ;

- le débriefing porte sur les situations vécues. Il est conduit par les formateurs, généralement sur le principe d'échanges réflexifs et constructifs. Dans les situations de débriefing observées, les formateurs conduisent ce débriefing sans qu'il y ait un protocole prédéterminé, ils cherchent à s'appuyer sur les objectifs pédagogiques du scénario.

\section{Présentation du plan de l'article}

L'étude prend comme objet la formation des opérateurs en anesthésie-réanimation (MAR, IADE) aux compétences non-techniques. Cette formation, comme indiqué précédemment, est construite sur la mise en scène des deux corps professionnels afin de rester au plus près de la réalité. Il est bien évident que chaque opérateur a des missions et des fonctions différentes. Cependant, au quotidien ils les exercent en coprésence, ce qui détermine la nécessité de développer par la simulation des compétences non-techniques transversales aux deux corps professionnels (verbalisation des actes, des informations techniques, des difficultés perçues, coopération, travail en équipe...). Mais il s'agit également de développer des compétences non-techniques spécifiques relevant du MAR (planification, prise de décision, allocation des tâches, leadership...). Nous remarquons ainsi que c'est justement cette coprésence qui permet la mise en oeuvre des compétences non-techniques.

Le premier temps de notre programme de recherche, qui est l'objet du présent article, relève d'une approche exploratoire qui a pour objectif d'observer et d'analyser l'activité des formateurs, les codes professionnels et les jeux de langage... Dans un second temps, un dispositif de formation de formateurs centré sur le débriefing sera construit et expérimenté. Dans un troisième temps, ce dispositif fera l'objet d'une évaluation.

L'analyse des compétences non-techniques et de la situation de débriefing prend appui sur des éléments théoriques issus notamment de la didactique professionnelle (Pastré, 2002 ; 2005 ; 2011) et des propositions de Chiniara et Pellerin (2014) que nous exposons (paragr. Éléments de cadrage théorique). Nous présentons alors le dispositif de recueil de données : observation des situations de formation et de débriefing (paragr. Méthodologie : recueil et modalités d'analyse des données). Nous analysons ensuite ces données (paragr. Résultats et analyse des données). Nous proposons enfin, de manière heuristique, des éléments visant à faire évoluer les situations de débriefing de manière à rendre plus efficace la formation aux compétences non-techniques (paragr. Conclusion et perspectives : des pistes pour une formation de formateurs).

Le premier temps de notre programme de recherche, qui est l'objet du présent article, relève d'une approche exploratoire qui a pour objectif d'ob- 
server et d'analyser l'activité des formateurs, les codes professionnels et les jeux de langage... Dans un second temps, un dispositif de formation de formateurs centré sur le débriefing sera construit et expérimenté. Dans un troisième temps, ce dispositif fera l'objet d'une évaluation.

\section{ÉLÉMENTS DE CADRAGE THÉORIQUE}

De nombreux auteurs (Perrenoud, 1997 ; Vergnaud, 1990 ; Samurçay \& Pastré, 1995 ; Bronckart, 2009 ; Pastré, 2011) notent fort justement la polysémie du terme " compétence ». Il ne s'agit pour nous ici ni de discuter de ce concept, ni d'ajouter une nouvelle définition. Nous insérons nos réflexions de manière naturelle et écologique dans les approches qu'utilisent, au plan international, les chercheurs (Anceaux \& Beuscart-Zéphir, 2002 ; Reader, Flin, Lauche, \& Cuthbertson, 2006) impliqués dans des problématiques proches des nôtres : la formation de professionnel de la santé. Cette approche paraît adaptée, au moins dans le premier temps de notre programme de recherche, pour l'étude exploratoire des débriefings de formation.

Nous distinguons en particulier, d'une part les compétences techniques liées aux savoirs et aux savoir-faire expérientiels (savoir agir) et d'autre part les compétences non-techniques qui regroupent ce qui est parfois décliné en savoir participer (coopérer, collaborer, organiser) et en savoir-être. Ces compétences non-techniques sont parfois désignées comme des compétences transversales (Riem, Boët \& Bould, 2012 ; Jaffrelot \& Savoldelli, 2013 ; Verscheure, 2015).

Distinguer ces deux types de compétences ne sous-entend pas, selon nous, qu'il faille les penser indépendantes. Il est bien évident que, par exemple, une communication entre professionnels en bloc relève à la fois de compétences techniques (des savoirs) et de compétences non-techniques (gestion des interactions). Nous suivons ici Riem et al. (2012) qui confirment l'existence d'une relation entre elles, même si cette relation demande encore investigation. L'accent mis ici sur les compétences non-techniques peut être certainement vu aussi comme conséquence d'un souhait formulé par la Haute Autorité de la Santé (HAS, 2012) et d'une demande des institutions impliquées dans nos travaux (centres de formation dans les hôpitaux universitaires).
Les compétences non-techniques dans la formation des professionnels de santé : de quoi parle-t-on?

Dès les années soixante, selon Cooper \& Gaba (2002) l'anesthésie a porté intérêt aux raisons et aux conséquences de ses erreurs afin de tenter de les réduire et de mieux les gérer. Les travaux de Gawande, Zinner, Studdert \& Brennan (2003) ont mis en évidence que dans $43 \%$ des cas, ce sont des problèmes de communication et de dysfonctionnements dans les équipes qui généraient des erreurs médicales; et de nombreuses études ont montré l'importance des facteurs humains dans la gestion d'une anesthésie (voir par exemple la méta-analyse de Cooper et al., 2002). Les réflexions et les préconisations ont alors permis de réduire le taux de mortalité directement lié à l'anesthésie. Celui-ci est passé de 1 décès sur 13200 cas en 1980 à 1 sur 140000 en 1999 en France (Lienhart, Auroy, Pequignot \& Benhamon, 2006).

Cependant Neyns (2011) estime encore à 80000 le nombre de cas de complications graves en France par an (et à 14 millions dans le monde) et selon la société française d'anesthésie et de réanimation (2010), 20000 à 40000 décès par an en France pourraient être évités.

De nombreux facteurs humains (individuels ou collectifs) sont aujourd'hui étudiés : la gestion et l'organisation de la tâche (p. ex. Von Wyl, Zuercher, Amsler, Walter \& Ummenhofer, 2009), le travail d'équipe (p. ex. Naik \& Brien, 2013), la maîtrise des émotions (Geeraerts, 2009), le leadership (p. ex. Cooper, 1999), la prise de décision (p. ex., Chiniara \& Pellerin, 2014), la sécurité psychologique (Chiniara \& Pellerin, 2014), la conscience de la situation (à partir de Gaba, 1995).

Deux apports sur les compétences non-techniques nous semblent à souligner, la conscience de la situation qui a fait l'objet de nombreux travaux que nous reprenons ci-après et la dichotomie opérée par Chiniara et Pellerin (2014) à propos des compétences non-techniques. Ces derniers considèrent que les unes sont liées aux habiletés cognitives et les autres aux habiletés interpersonnelles ainsi que les principes à l'œuvre dans chaque cas. 


\section{La conscience de la situation}

Endsley (2011) reprend cette dernière proposition, il note l'importance du développement de cette conscience, particulièrement dans des conditions critiques. Le collectif professionnel doit avoir une représentation partagée de la situation dans laquelle il est engagé. Pour Fioratou, Flin, Glavin et Patey (2010) également, la mise en ouvre in situ et in vivo de cette conscience de la situation constitue une des compétences non-techniques fondamentales dans la perspective d'une pratique d'anesthésie sécuritaire.

Selon Schulz, Endsley et Kochs (2013), la conscience de la situation se déploie à trois niveaux : la perception des informations; la compréhension de ces informations par rapport à l'état du patient et aux données sous-jacentes; la projection ou l'anticipation de l'évolution de l'état du patient pour la gestion proactive d'une situation dynamique, c'est-à-dire la prise de décisions et l'action.

$\mathrm{Au}$ premier niveau, cette modélisation repose donc sur la perception et la sélection des informations par les professionnels. La situation étant dynamique, chaque professionnel met en ouvre des actions qui ont une incidence sur le déroulement même de la situation globale. Ces actions et ces situations nécessitent en permanence, pour être conduites de manière efficiente, des processus d'adaptation individuels et collectifs. Cette variabilité situationnelle dépend des actes chirurgicaux (nature, durée, équipements, instruments...), de la singularité du patient, de l'équipe en présence (variable d'une intervention à une autre), des produits utilisés (en fonction du patient et de l'intervention), des aléas de l'intervention.

Ainsi, l'activité de l'équipe d'anesthésie (MAR et IADE) est fondée sur une forme d'anticipation car les causes d'incidents sont multiples et complexes (Sheridan, 2008). Des travaux de recherche ont mis en évidence que la conscience de la situation peut être altérée par le biais d'ancrage (Tversky \& Kahneman, 1974) qui consiste à fixer l'action sur une évaluation hâtive, le biais de confirmation (Croskerry, 2009) qui correspond à un choix d'informations uniquement orientées par l'hypothèse première, ou encore la cécité cognitive liée à la difficulté à détecter tous les changements dans une situation complexe et dynamique. La conscience de la situation et les biais qui peuvent l'altérer sont potentiellement présents quel que soit le corps professionnel. Par exemple, le MAR et le IADE peuvent dans un même élan ne repérer ou ne rechercher que les paramètres physiologiques concordants avec le diagnostic médical (tension artérielle, température, alarmes...).

La salle d'opération correspond donc à un environnement complexe et dynamique dans lequel plusieurs groupes professionnels (chirurgiens, anesthésistes, infirmiers...) d'où la nécessité d'une communication globale efficiente. Naik et Brien (2013) confirment que la sécurité du patient, notamment en situation de crise, repose sur une synergie mettant en œuvre des compétences techniques et des compétences non-techniques de tous les professionnels.

\section{Les habiletés cognitives et les habiletés interpersonnelles}

Chiniara et Pellerin (2014) proposent de définir les habiletés non-techniques à partir de deux registres : les habiletés cognitives, plutôt liées à chacun des professionnels-individus, et les habiletés interpersonnelles, plutôt liées aux aspects sociaux, en situation. Ils opérationnalisent ensuite chacune de ces habiletés au regard de principes liés à des actions ou à des positionnements relatifs à l'organisation de la situation (cf. tableau ci-après). On retrouve une approche semblable chez Pastré (2011) lorsqu'il prend l'exemple de la conduite simulée de centrales nucléaires et qu'il associe les compétences non-techniques aux nécessités d'adaptation dans un milieu complexe (variabilité des situations, équipe de professionnels).

Il nous semble :

- que cette proposition peut être perçue comme une synthèse des approches des compétences non-techniques (liées aux facteurs humains) précédemment évoquées, complétée par des éléments pertinents au regard de l'analyse des situations projetées (émotion, responsabilité, climat de confiance);

- d'autre part, que les mises en perspectives de ces habiletés, à la fois d'un point de vue individuel et du point de vue du collectif engagé, permettent de faire fonctionner, de manière heuristique, le modèle de la conscience de la situation. 
Tableau 1.

Habiletés cognitives et interpersonnelles (d'après Chiniara et al., 2014)

\begin{tabular}{|c|c|}
\hline Habiletés cognitives & Principes \\
\hline Planification & $\begin{array}{l}\text { Gestion efficace des ressources humaines et matérielles } \\
\text { Anticipation des besoins } \\
\text { Priorisation des tâches }\end{array}$ \\
\hline Prise de décision & $\begin{array}{l}\text { Responsabilité du leader } \\
\text { Décisions dynamiques et réfléchies } \\
\text { Décisions basées sur une connaissance complète de la situation }\end{array}$ \\
\hline Allocation des tâches & $\begin{array}{l}\text { Distribution des tâches selon les forces de chacun } \\
\text { Plasticité des rôles }\end{array}$ \\
\hline Sensibilité situationnelle & $\begin{array}{l}\text { Analyse continue de la situation } \\
\text { Surveillance de l'efficacité des interventions } \\
\text { Anticipation de l'évolution du patient } \\
\text { Partage d'un modèle mental commun } \\
\text { Résumés fréquents et synthèses intermédiaires }\end{array}$ \\
\hline Habiletés interpersonnelles & Principes \\
\hline Communication & $\begin{array}{l}\text { Transmission d'un message de façon claire, précise et assertive } \\
\text { Échange d'informations centré sur le leader } \\
\text { Coordination des tâches }\end{array}$ \\
\hline Leadership & $\begin{array}{l}\text { Désignation explicite du leader } \\
\text { Identification des objectifs } \\
\text { Définition des rôles de chacun } \\
\text { Recueil de l'information } \\
\text { Prise de décisions cliniques }\end{array}$ \\
\hline Travail d'équipe et synergie de groupe & $\begin{array}{l}\text { Accomplissement des rôles de chacun } \\
\text { Double surveillance au besoin } \\
\text { Coopération lorsque nécessaire } \\
\text { Tâches orientées vers des objectifs communs } \\
\text { Résolution des conflits de façon constructive } \\
\text { Surveillance des autres membres de l'équipe et du leader }\end{array}$ \\
\hline
\end{tabular}

\section{Le débriefing : pourquoi ?}

« Malgré la place indiscutable accordée au débriefing dans différentes productions scientifiques - les données analysées de 109 articles présentent le débriefing comme une étape essentielle (Granry \& Moll, 2012) - nous soulignons le peu d'études portant sur les caractéristiques, le processus et l'impact du débriefing dans la formation médicale » (Bastiani, Calmettes \& Minville, 2016).

\section{Les apports de la didactique professionnelle}

Le champ de la didactique professionnelle nous paraît pertinent en termes de référence théorique car il permet « de prendre en compte l'ensemble des composants de la compétence professionnelle [...], d'analyser les déterminants de son développement et de proposer la conception de situations de forma-
tion»(Rogalski, 2004, p. 103). Et l'objectif de notre programme de recherche est bien de repérer les éléments qui fondent la professionnalisation et le développement professionnel des formateurs par l'élaboration projetée d'une formation de formateurs.

En didactique professionnelle, le débriefing est perçu comme une étape fondamentale de la formation. Selon Pastré (1999), c'est « à ce moment-là que s'opère la conceptualisation de la situation sous sa forme pragmatique et que les acteurs découvrent, après coup, avec le sens de leurs erreurs, l'articulation entre équilibres de base, indicateurs et régimes de fonctionnement». C'est la compréhension du sens de ce qui a été fait qui permet de construire le modèle pragmatique pour l'action. L'analyse du débriefing permet de construire une dialectique entre une réalité professionnelle vécue (la conduite du débriefing) et une évolution / reconstruction de celle-ci par la formation. 
Généralement l'écart entre tâche et activité constitue le point de départ du travail d'analyse. Selon Pastré, Mayen et Vergnaud (2006), pour identifier la structure conceptuelle d'une situation, ici par le débriefing, il est nécessaire de repérer les invariants mobilisés chez les sujets ayant une action efficace. Pastré (2005, p. 243), précise que cette structure conceptuelle est élaborée à partir de trois composantes : « les dimensions extraites du réel dans sa globalité [...], les observables, [...] les classes de situations ».

Nous faisons l'hypothèse que l'approfondissement de l'analyse grâce au cadre théorique des compétences non-techniques tel que décrit ci-dessus est heuristique pour la construction des observables et donc de la structure conceptuelle de la situation. Les habitudes cognitives et interpersonnelles et les principes associés peuvent participer à la construction des concepts organisateurs de l'action. Pastré (2002, p. 15) souligne qu'en situation dynamique ces concepts « multiples [...] forment un réseau [et c'est] ce qui amène à parler d'une structure conceptuelle de la situation ».

Nous poursuivons à partir des travaux réalisés sur les débriefings dans les formations des professionnels de santé.

\section{Le débriefing dans les formations de santé}

Le débriefing a pour objectif d'analyser les actions des formés, les connaissances qu'ils mettent en jeu, les attitudes et les ressentis, afin de proposer des axes d'évolution de pratiques. On considère ici que le débriefing «ne [porte pas] uniquement sur les résultats (outcome) de la simulation, mais surtout sur la qualité des processus (process) et des comportements [...]. Autrement dit, [le débriefing] s'intéresse davantage à la question : pourquoi tel résultat est survenu ? Qu'à la question : quel résultat est survenu ?»(Chiniara \& Pellerin, 2014, p. 376). Autrement dit, l'enjeu de la formation est le repérage et la compréhension des événements.

Le débriefing est largement utilisé dans la formation des professionnels de santé (voir la méta-analyse de Granry \& Moll, 2012), comme il l'est maintenant pour de nombreux autres milieux professionnels (aéronautique, ingénierie, enseignement, etc.). La place et le rôle des formateurs dans ces débriefings, ou la structuration et les contenus de ces débriefings font l'objet de nombreuses recherches.
Par exemple, Dismukes et Smith (2001) discutent du rôle du formateur dans la dynamique du débriefing et ils qualifient différents niveaux de débriefing au regard des postures des formateurs. Dans le niveau « haut », les participants ont déjà un bon niveau de réflexion et le formateur se positionne comme catalyseur pour que le groupe développe lui-même les solutions. Dans le niveau « bas », l'investissement personnel du formateur sera constant, plus directif, laissant peu d'initiative aux apprenants.

La structuration temporelle des débriefings est présentée en trois étapes par Zigmont, Kappus et Sudikoff (2011) : la phase de désamorçage (defusing) qui a pour objectif de neutraliser les effets de postures bloquantes liées aux erreurs perçues et à l'émotion, la phase de découverte ou de construction (discovering) qui permet le développement des échanges de manière ouverte et la phase d'approfondissement (deepening) qui conduit à la fois à des structurations et à des synthèses visant si nécessaire une évolution des compétences en jeu.

\section{MÉTHOdOLOGIE : RECUEIL ET MODALITÉS D'ANALYSE DES DONNÉES}

Le projet de recherche dans son ensemble a été validé par l'Institutional Review Board (IRB 00003888) du Comité d'Évaluation Éthique de l'Inserm Paris.

\section{Un premier recueil d'observations}

Nous avons réalisé 33 vidéos de débriefings (phase exploratoire) dans un Institut de Simulation en Santé. Six vidéos ont été exclues à la demande des formés ou pour des raisons techniques (perturbations sonores, niveau de parole trop bas) et cinq l'ont été en raison d'un temps de parole relatif aux compétences non-techniques inférieur à $2 \mathrm{~min}$. Il s'agit donc de l'analyse et de l'interprétation de 22 vidéos.

On relève que neuf vidéos présentent de fortes redondances, soit en raison de la présence d'un même formateur soit en raison de scénarios utilisés identiques ce qui est en faveur d'une certaine stabilité du processus de débriefing, ancré dans les pratiques quelle que soit la situation vécue. Pour construire les critères pour l'étude, nous avons observé les vidéos en relevant la présence ou non des conditions favorisant l'analyse de l'action (Pastré, 1999) : temps 
d'échanges et qualification des locuteurs. Un temps d'échange important et des échanges pluripersonnels permettent la conceptualisation de la situation sous sa forme pragmatique (Pastré, 2002).

Nous avons relevé les aspects quantitatifs relatifs à la part du temps donné aux compétences nontechniques. Nous nous sommes ensuite intéressés aux conditions organisationnelles du débriefing qui se déroulent avec 2 à 4 formateurs et 6 à 8 formés. Nous avons observé la posture du formateur en termes de facilitation de l'analyse de l'action du point de vue interactionnel (Dismukes $\&$ Smith, 2001). Nous avons aussi relevé les conduites communicationnelles mises en œuvre par les formés, allant de la stratégie d'évitement pour juguler la menace de l'estime de soi (déstabilisation, regard de l'autre, possibilité d'erreur...) jusqu'à la rencontre de l'obstacle à dépasser, car ces conduites font partie de la situation de formation et constituent pour partie la variabilité des situations (Pastré, 2002).

En lien avec les propositions de Chiniara (paragr. Les habiletés cognitives et les habiletés interpersonnelles), nous pouvons dire si les pratiques des formateurs - apports de connaissances, travail métacognitif, prise en compte des émotions, du stress, réassurance - permettent ou pas le développement des habiletés cognitives (planification, prise de décision, allocation des tâches, sensibilité situationnelle) par une prise de conscience du formé. Les éléments relatifs aux interactions et à la posture des formés peuvent eux aider ou pas au développement des habiletés interpersonnelles (communication, leadership et travail en équipe).

Cette analyse des données s'inscrit dans un paradigme compréhensif et vise à la fois la description des pratiques actuelles de débriefing et le résultat de la confrontation des formateurs à ces pratiques afin de comprendre comment ils vivent les débriefings, ce qu'ils veulent générer par leurs interventions auprès des formés et ce qu'ils pensent nécessaire d'améliorer.

\section{Un deuxième recueil avec des observations et des entretiens}

À l'issue de la première analyse, nous avons à nouveau réalisé des vidéos de débriefing suivies d'entretiens d'auto confrontation avec les formateurs: 30 vidéos et 16 entretiens. Nous avons choisi de faire des entretiens d'auto confrontation simple (Clot,
2000). Il s'agit d'amener le formateur, par le questionnement du chercheur, à verbaliser autour de ses pratiques, stimulé par la vidéo du débriefing réalisé. Ce questionnement s'intéresse au vécu de l'action du formateur aux niveaux émotionnel, conceptuel et procédural. La difficulté, pour le chercheur, réside dans le passage d'une description, d'énoncés généraux - « les médecins vont plus analyser ce qu'ils ont fait, les infirmiers analysent la situation en général » - à une véritable évocation, une parole incarnée « je suis en échec face au mutisme de l'étudiant, alors qu'est-ce que je fais? Je commets une erreur, j'occupe la parole pour me donner l'impression que j'apporte quelque chose " (MAR Formateur, vidéo 9) - ici le formateur parle au présent et il utilise le « je ». Dans ces moments d'évocation, nous constatons généralement chez le formateur une modification du débit de parole, plus lent, des propos qu'il s'adresse à luimême. Cette verbalisation déclenche une prise de conscience de la situation et permet le passage de l'action vécue à sa représentation.

Ces entretiens, réalisés dans les 48 heures après la captation vidéo, ont permis des verbalisations sur l'activité visible (ce que nous montre la vidéo) mais aussi sur l'activité invisible, c'est-à-dire sur les processus à l'œuvre chez le formateur dans la décision d'orienter de telle ou telle façon le débriefing, sur les attitudes et comportements vis-à-vis des formés dans le bloc, des autres formateurs et des observateurs. Cette parole réflexive produit des informations sur la conduite - « comment je conduis le débriefing »- et produit une conceptualisation par l'identification d'une structure conceptuelle de la situation vécue.

La confrontation à l'activité filmée a permis de revivre la situation par l'intermédiaire de l'image et de soutenir le retour mémoriel. Cette économie cognitive facilite l'accès aux aspects ressentis de l'activité, le sujet participe ainsi de manière active à l'entretien. Il peut se concentrer sur la dimension cognitive de ses actions en situation (discours, posture, comportement...) et sur les réflexions mentales qu'il a réalisées au cours de l'activité qui ne peuvent être restituées sans sa participation active dans l'entretien.

Nous devons signaler la nécessité, pour le chercheur, d'une connaissance approfondie des activités étudiées pour mener à bien ce type d'entretien. Celle-ci a été construite par une acculturation au terrain (immersion au bloc opératoire, assistance aux enseignements des formés, accompagnement d'une 
équipe médicale aux urgences) - sous la supervision d'un praticien hospitalier, professeur des universités - et à partir de l'analyse des vidéos de la phase exploratoire.

\section{RÉSULTATS ET ANALYSE DES DONNÉES}

Pour des débriefings qui durent de 16 à 46 min, le temps d'échanges en rapport avec les compétences non-techniques va de 2 min (sur 15 min de débriefing) à 20 min (sur 46 min de débriefing); celui concernant les compétences techniques est de 7 min (sur 10 min de débriefing) à 40 min (sur 45 min de débriefing). Nous constatons un temps de parole technique / non-technique étroitement lié à la durée du débriefing avec une prépondérance pour les aspects techniques quelle que soit la durée du débriefing, avec certes de bonnes raisons de revenir sur les compétences techniques. Les formateurs y occupent majoritairement le temps de parole (60 à $90 \%$ du temps).

À partir de la grille de Chiniara (paragr. Éléments de cadrage théorique) associée aux focales d'analyse des vidéos (paragr. Méthodologie : recueil et modalités d'analyse de données) nous constatons que les différents aspects des compétences non-techniques ne sont que partiellement repris dans les débriefings. Les formateurs prennent parfois conscience de ce fait dans les entretiens.

\section{Les habiletés cognitives}

\section{Observation des vidéos}

Pour ce qui relève des habiletés cognitives il s'agit d'amener les formés à un certain niveau de réflexivité (métacognition) autour des différents processus mis en œuvre.

Nous pouvons noter, concernant la planification, que le débriefing de cette compétence se présente dans une seule vidéo. Un formé exprime « je ne me suis pas concentré sur le diagnostic, je me suis centré sur l'exécution à fond » (Formé Infirmier, vidéo 12), ce qui donne lieu à une réflexion "ça me choque pas que tu te centres sur la tâche » (IADE Formateur, vidéo 12) et nous constatons que le formateur engage l'échange pour amener les formés à voir l'intérêt de comprendre la situation quand il y a beaucoup de tâches afin de les prioriser. Le formateur amène un autre formé qui certainement rationalise en disant « j'étais obligé d'être dans l'action parce qu'il y avait beaucoup de choses à faire " (Formé Médecin, vidéo 12) à prendre conscience qu'il avait à sa disposition l'aide nécessaire pour accomplir les tâches. Nous remarquons dans certains cas que le formateur retire une possibilité de réflexion au formé par une affirmation immédiate relative à l'anticipation des besoins « pourquoi t'as pas appelé à l'aide plus tôt » (MAR Formateur, vidéo 9).

Nous notons différentes approches qui relèvent de la prise de décision. Elles dénotent soit une volonté de conduire les formés à réfléchir sur leurs actes « d'autres voies sont possibles pour fonder votre décision » (MAR Formateur, vidéo 7), soit un renversement de situation. Dans ce cas, le formateur fait part de la réflexion qu'il suppose avoir été menée par les formés, afin de trouver une solution alternative « là tu as fait ça parce que tu pensais...» (MAR Formateur, vidéo 6) ou « moi face à l'hyperthermie maligne j'aurais refait la check-list » (MAR Formateur, vidéo 3) devançant ainsi toute possibilité réflexive chez les formés.

L'allocation de tâches est un point rarement travaillé. Les formateurs tentent parfois de faire évoluer les points de vue des formés « une IBODE (infirmière de bloc) reste une infirmière, elle peut préparer l'adrénaline, une aide-soignante comme tout personnel hospitalier a fait une formation aux soins d'urgence donc elle peut masser » (MAR Formateur, vidéo 10) mais ce type d'assertion n'est pas dans une intention réflexive mais plutôt de l'ordre d'une affirmation.

Pour ce qui relève de la sensibilité situationnelle, un formateur souligne que la gestion de tâches en situation de crise implique que « le teamleader ne doit pas faire le massage cardiaque car il serait fixé sur sa tâche qui est complexe et ne pourrait plus avoir l'œil partout » (MAR Formateur, vidéo 3) ou encore que «faire quelque chose c'est contra phobique ${ }^{2}$ mais il faut que quelqu'un ait le recul nécessaire pour anticiper » (MAR Formateur, vidéo 3). Nous sommes toujours ici dans le registre de l'affirmation qui rompt l'interaction entre formés autour de la place de chacun dans le scénario. 


\section{Étude des entretiens}

Ces éléments associés aux entretiens d'auto confrontation nous amène à souligner qu'il serait nécessaire de minorer les apports directs de connaissances. Les formateurs constatent qu'ils devraient saisir ou provoquer davantage les opportunités de travail sur les compétences non-techniques. Ils relèvent ainsi qu'ils interviennent davantage sur des apports de connaissances, et qu'ils favorisent la « correction » de l'exercice plutôt que la réflexion sur l'action. Certains utilisent des supports. Celui-ci comporte essentiellement des apports de connaissances : " c'est comme si on leur donnait un cours, on leur donne des critiques, vous avez fait ça, ou pas fait ça » (MAR Formateur, vidéo 6). Les formateurs utilisent parfois un support qui vise à illustrer une situation non-technique mais l'exploitation de celuici n'est pas ou est peu vecteur de réflexivité chez les formés : « je passe la vidéo mais je ne la travaille pas vraiment avec eux, dommage... je me rends compte que cet exemple pourrait vraiment déclencher une discussion par analogie avec la simulation vécue » (IADE Formateur, vidéo 1).

Si les formateurs usent de formulations majoritairement positives, les interactions avec les formés sont principalement à base d'apports cognitifs sur un mode de transmission : « je suis sympa dans mon approche, le but n'est pas de les enfoncer, je ne veux pas qu'ils perdent confiance mais bon... je ne sais que dire comment il fallait faire techniquement » (MAR Formateur, vidéo 4). Les formateurs relèvent ainsi la quasi-absence de travail sur les processus à l'œuvre dans les décisions des formés (métacognition). Ils proposent, aux fins de remédiation, une répartition des missions formatrices sur deux personnes, avec deux rôles différents : " un rôle d'expert technique parce que je pense qu'il y a une demande là aussi des participants et puis peut-être quelqu'un d'autre qui va être plus sur les interactions (non-techniques) dans le cadre de la simulation» (MAR Formateur, vidéo 3). Ils conviennent qu'il est nécessaire de laisser de côté leurs propres réflexions sur le scénario afin que la réflexion des formés passe au premier plan : « je peux avoir une idée, un avis mais je vais essayer de faire cheminer une réflexion chez les participants » (IADE Formateur, vidéo 2), « je dois laisser la place aux apprenants, ce que je pense de la situation n'est pas essentiel... je suis trop présent » (MAR Formateur, vidéo 1).

\section{Les habiletés interpersonnelles}

\section{Observations}

Du point de vue des habiletés interpersonnelles, le but ici est de repérer et de discuter des actions en lien avec les aspects communicationnels et le positionnement des acteurs.

En termes de communication, si la question en jeu dans la situation est fréquemment évoquée, nous retrouvons des formes plutôt affirmatives limitant tout échange : « se forcer en deux trois mots-clés à décrire la situation » (IADE Formateur, vidéo 12) ou encore « un homme, une mission, le closeout : faire l'acte et faire remonter l'information que c'est fait » (IADE Formateur, vidéo 4). En conséquence, rien n'est dit sur la prise de conscience de cette situation par les formés.

Le leadership est également abordé par les formateurs, avec des formulations de type " il est nécessaire de se détacher pour assurer le leadership de la situation, il faut pour avoir une bonne vision d'ensemble se détacher du côté technique » (MAR Formateur, vidéo 12) ; « le renfort est arrivé et a pris le teamleader, c'est une question de caractère » (MAR Formateur, vidéo 4) ; « aux États-Unis, le teamleader se met aux pieds, il a sa check-list, il donne les ordres et regarde l'heure » (IADE Formateur, vidéo 3). Ces interventions sont réalisées sans que les formés puissent discuter.

Pour le travail d'équipe et la synergie de groupe, les propos laissent émerger les représentations de certains formés «si le médecin pose un diagnostic moi j'exécute » (Formé Infirmier, vidéo 9), ce qui éclaire sur leur positionnement d'attente. Dans les autres vidéos, les formateurs reviennent majoritairement aux assertions sous une forme injonctive : « ce n'est jamais celui qui a intubé qui ausculte » (MAR Formateur, vidéo 12) ; « un infirmier anesthésiste, il sait intuber par exemple, tu fais confiance, tu laisses faire mais la confiance n'exclut pas le contrôle * (MAR Formateur, vidéo 10).

\section{Éléments d'analyse}

Les axes identifiés par Chiniara se retrouvent au travers des différentes vidéos de débriefing analysées mais il apparaît que leur exploitation reste partielle 
et sous une forme qui limite la prise de conscience rétrospective chez les formés.

Ainsi, il s'agirait pour les formateurs de penser des objectifs relatifs à la construction des compétences non-techniques. Le poids important des compétences techniques dans les échanges amène les formateurs à s'interroger sur la nécessité d'une formalisation des objectifs liés aux compétences non-techniques en amont du scénario de crise, lors du choix du cas étudié en simulation : « je me donne l'illusion que les compétences non-techniques sont pas au premier plan des objectifs mais en réalité c'est le cas » (MAR Formateur, vidéo 9). Nous constatons que les scénarios sont majoritairement construits autour d'exceptions professionnelles qui génèrent une situation de crise au bloc opératoire. Ils ne sont pas pensés en fonction d'objectifs pédagogiques en lien avec les compétences non-techniques. « Comme si les compétences non-techniques devaient découler naturellement de ces situations » (MAR Formateur, vidéo 1).

Il s'agirait également de valoriser des démarches collectives. Sans diminuer la valeur primordiale du diagnostic, il apparaît que la valorisation du bon diagnostic peut générer des démarches solitaires de la part des participants : " parfois les internes privilégient leur propre réussite dans la découverte du diagnostic, ils ne communiquent pas et se lancent dans l'action » (IADE Formateur, vidéo 5). Ces attitudes entrent en conflit avec l'objectif de développement des compétences non-techniques et notamment de la communication dans l'équipe : " pour moi, la simulation c'est surtout mettre en avant les interactions dans une situation de crise " (MAR Formateur 8). La nécessité de verbaliser, si elle est minorée, peut générer un risque de biais de centration du médecin. Nous relevons que le débriefing est nettement plus court lorsqu'il y a un haut niveau de réussite du scénario, d'un point de vue technique, par les formés. Un formateur constate que fréquemment dans ces cas-là « on a un superviseur qui valide des choses en disant, je suis d'accord, je valide » tout en précisant qu'il n'est pas favorable à cette façon de faire : « je pense que c'est pas du tout une approche appropriée » (IADE Formateur, vidéo 1) et qu'un échange serait nécessaire.

Nous comprenons ici que le débriefing est à adapter aux stratégies des formés. Dans certains cas, nous constatons que, malgré un temps de parole des formés supérieur à celui des formateurs, les échanges sur les aspects techniques sont majoritaires. Les formés parlent du domaine dans lequel ils sont le plus à l'aise et évitent d'aborder les aspects non-techniques sur lesquels ils pourraient être en difficulté, ils adoptent des stratégies d'évitement. Ce serait au formateur de réguler les points abordés. Mais face à la résistance des formés à s'exprimer et à parler de leur ressenti en " se cachant» derrière la technique: « je trouve qu'on s'en est bien sorti, je préfère parler des aspects techniques » (Médecin Formé, vidéo 10), le formateur se sent bloqué : « je les vois qui esquivent, qui se jettent à corps perdu dans le technique, j'avoue que je ne sais pas trop comment les ramener au non-technique parce que je ne suis moi-même pas très à l'aise avec cela, donc ça m'arrange " (MAR Formateur, vidéo 2). Les formateurs constatent qu'il n'y a pas de moments de silence, mais une « occupation » du temps (discussions diverses extérieures au cas ou plaisanteries) autant du côté des formés pour éviter de parler de leur limite en termes de réactivité technique que du côté des formateurs pour éviter de stigmatiser l'échec par exemple.

Par ailleurs, des interactions entre les formés sont à développer. Les formateurs reconnaissent que les interactions actuelles occultent souvent les possibilités d'introspection ou de réflexion de la part et avec les formés « j'aurais dû beaucoup moins parler, laisser un temps de parole beaucoup plus important à ces participants et j'aurais dû essayer de faire en sorte qu'ils arrivent à une compréhension entre eux de ce qui s'est passé, de ce qui s'est joué, du phénomène vécu » (MAR Formateur, vidéo 1).

Les observateurs doivent être impliqués. Les formateurs prennent conscience de la faible implication des observateurs, qui paraissent passifs ou absents. Ils émettent l'idée de repenser leur rôle, « on sent qu'il y a des aller-retour entre eux [les formés qui ont participé au scénario] ; la parole n'est pas vraiment donnée à tout le monde [les observateurs] » (MAR Formateur, vidéo 4). Ce rôle pourrait être repensé à partir du fait que les observateurs constituent un renfort qui peut être sollicité lors de la simulation « la passivité des observateurs doit être une préoccupation, c'est le formateur qui est responsable de cette passivité " (IADE Formateur, vidéo 2).

Les éléments de langage, point nodal des interactions notamment en situation de crise, nécessiteraient selon les formateurs des développements afin notamment de mettre en évidence la pertinence d'expressions claires et précises : « dire à l'aide-soignante, j'ai besoin d'un anesth, n'est pas forcément clair, il 
faut lui dire précisément va chercher d'urgence un médecin anesthésiste » (MAR Formateur, vidéo 2).

La question du leadership doit être travaillée, notamment dans les situations où un changement de leadership est opéré, ce qui génère un flottement handicapant pour le déroulement de la situation d'autant plus qu'il peut être relativement long.

\section{Des conditions d'exercice à réinterroger}

Au-delà des interrogations autour de la pratique de débriefing, les formateurs évoquent des éléments qui relèvent plus largement de l'organisation de l'équipe pédagogique.

\section{Une équipe pédagogique à former et une image à modifier}

Nous constatons la présence d'un grand nombre de formateurs dont les interventions varient en termes de temps consacré dans l'année à la simulation. Certains éprouvent des difficultés à reprendre une posture (rarement pratiquée) à chaque intervention et indiquent que par facilité ils ont tendance à aller vers les apports techniques (cognitif) : « je vois que j'ai du mal à me mettre dans le bain, je rame un peu, alors je me réfugie dans du technique » (MAR Formateur, vidéo 7). Ils soulignent que la référence à une compétence connue (compétences techniques) et le besoin de légitimation les amènent parfois à faire des apports conformes à la perception qu'ils ont de la situation sans tenir compte de la perception éventuellement différente que peuvent avoir les participants. Certains formateurs disent se référer principalement aux apports techniques en raison d'une forte expérience reconnue par leurs pairs et par les formés : « c'est ce qu'ils attendent de moi » (MAR Formateur, vidéo 4).

\section{Une concertation pédagogique à organiser}

Les formateurs (2 à 4), ne se concertent pas avant la séance. Ils évoquent une vision individuelle du débriefing par absence de concertation : « on n'avait pas vraiment organisé comment on allait faire ce débriefing $[\ldots]$ on ne savait pas qui allait être les formateurs présents » (IADE Formateur, vidéo 14).
Ceci génère des débriefings polymorphes avec des structures très différentes tant sur la durée que sur le contenu.

La variabilité des débriefings est liée également à l'adaptation aux développements inattendus d'une séance, mais l'analyse entre travail prescrit et travail réel n'est pas approfondie. Les formateurs évoquent également leur propre malaise « tout au fil du débriefing, je pense qu'on n'a pas trouvé notre place » (IADE Formateur, vidéo 1). Ils reconnaissent alors la nécessité d'un travail en amont dans l'équipe de formateurs du jour.

Une proposition est émise concernant le retour vidéo des scénarios : " utiliser les vidéos par exemple pour qu'ils se voient travailler » (MAR Formateur, vidéo 2) serait à la fois intéressant pour les formés mais aussi pour les formateurs dans le sens où les aspects non-techniques pourraient apparaître à nouveau, ce serait la mémoire de ces aspects occultés par la prééminence de la technique durant le débriefing.

Les formateurs soulignent que lorsque le scénario a été conçu par l'un des formateurs qui mène le débriefing, son regard est plutôt dirigé vers les attendus techniques : " je vois bien que lorsque je suis auteur du scénario j’attends ce que je sais déjà, [... je ne les aide pas à prendre conscience de ce qu'ils ont fait, je suis trop centré sur la correction. Cette question doit être discutée avec l'équipe pédagogique » (MAR Formateur, vidéo 4). Là encore, la vidéo serait utile car ce qui est valorisé, c'est le fait de trouver le bon diagnostic (technique). Pourtant, le fait de réussir le cas médicalement ne devrait pas exonérer de traiter les aspects non-techniques qui conduisent à élaborer ce bon diagnostic : " la découverte du bon diagnostic n'exonère pas de la discussion et pourtant... je me dis ils ont trouvé c'est bien et je conclus vite pour passer à la simulation suivante » (MAR Formateur, vidéo 3).

\section{Une progression pédagogique à adapter au niveau des formés}

Dans certains cas le scénario est très technique, par exemple sur une pathologie très rare qui ne peut pas être appréhendée par des médecins en début de cursus : " je repère que j'arrive à mettre les apprenants en échec simplement parce que le scénario choisi n'est pas conforme à leur niveau, c'est grave...» (MAR Formateur, vidéo 3). 
Les mêmes participants sont présents sur une journée et sont acteurs ou observateurs de différents scénarios, cependant les formateurs pensent que si les scénarios doivent être adaptés au niveau des participants, il faudrait aussi penser une progression pédagogique des scénarios sur une même journée : « j'ai conscience qu'il reste encore du travail sur la progression pédagogique d'une journée, c'est encore loin des objectifs premiers trop centrés sur le technique » (IADE Formateur, vidéo 5).

Le statut d'étudiant dans lequel sont les internes, dans leur activité réelle, a un impact sur la cohérence de leur statut par rapport aux scénarios dans lesquels ils sont considérés comme des séniors : «j'attends de l'interne qu'il se conduise comme un sénior expérimenté, je sais que ça ne va pas, ils ont droit à l'erreur, c'est à cela que sert la simulation » (MAR Formateur, vidéo 6).

\section{Le rôle et la place du formateur à définir}

La place des différents formateurs (médecins et infirmiers) est questionnée notamment par les infirmiers anesthésistes qui ont le sentiment que dans la simulation ils se retrouvent dans le même rapport qu'au bloc opératoire avec le médecin alors qu'à la simulation ils sont engagés dans la même posture de formateur : " au bloc dès qu'il y a un problème technique le médecin va voir l'infirmier [...] à la simulation, on évolue dans un environnement technique avec à la fois vidéo, audio, informatique, mannequin et là $[. .$.$] brancher les caméras, diriger le robot,$ configurer les scénarios, le reconditionnement du matériel, la place indéniable de l'infirmier elle est sur ce créneau » (IADE Formateur, vidéo 2). Le leadership se retrouve dans le débriefing, le médecin prend davantage de place que l'infirmier. Lorsque c'est le médecin qui parle le premier, la parole de l'infirmier est limitée « je ne me sens pas légitime quand le médecin-formateur parle, j'ai l'impression d'être inutile, de trop » (IADE Formateur, vidéo 6).

\section{La question évaluative à discuter}

Le sentiment de jugement notamment chez les formés infirmiers (qui sont des professionnels expérimentés venant dans le cadre de la formation continue) et leur place comme « exécutant » sont très présents dans certains discours. C'est un frein à la parole libérée attendue dans un débriefing : "j’ai remarqué à plusieurs reprises que les IADE (Infirmiers) sont en retrait dans le débriefing mais aussi des fois au cours du scénario, ils se sentent évalués, ils ont peur du regard porté sur leurs pratiques » (IADE Formateur, vidéo 3 ).

Une différence importante peut être notée entre la posture des internes et celle des infirmiers. Les internes semblent vivre les séances de simulation comme une partie de leur formation au même titre que les séminaires, ils sont dans une posture d'étudiants. Les propos des infirmiers, qui sont déjà des professionnels en fonction, laissent supposer qu'ils vivent l'expérience de la simulation plutôt comme un contrôle, une évaluation de leurs compétences.

\section{CONCLUSION ET PERSPECTIVES : DES PISTES POUR UNE FORMATION DE FORMATEURS}

La démarche de recherche, notamment grâce aux entretiens d'auto-confrontation, a amené les formateurs à s'interroger sur leurs pratiques et les a, d'une certaine manière, engagés dans le changement de ces pratiques. Nous retrouvons ici les propos de Pastré (2006) lorsqu'il souligne que la « réflexivité [sur l'action, qu'elle] s'exprime en cours d'action ou simplement après coup, [est] une des conditions [...] pour que l'activité constructive engendre du développement $»$ (p. 157).

Tout au long des commentaires que les formateurs ont émis, nous avons repéré une remise en question de leurs pratiques et la nécessité :

- d'une part de changer leur posture : ne pas faire un cours (type magistral) et créer les conditions de réflexivité chez les acteurs de la simulation ;

- d'autre part, d'harmoniser leurs pratiques, de se concerter autant au moment de la construction des scénarios que pour les débriefings.

Ils constatent aussi que leur seule légitimité technique ne peut pas remplir tous les attendus liés à la formation par la simulation.

Nous pouvons dire que si l'analyse des captations vidéo met en évidence l'activité productive des formateurs lors des débriefings, les entretiens d'autoconfrontation quant à eux représentent une première étape du processus d'activité constructive. Les verba- 
tim des formateurs montrent à plusieurs reprises une prise de conscience; par exemple « je me réfugie dans du technique »; « je ne les aide pas à prendre conscience de ce qu'ils ont fait, je suis trop centré sur la correction $», ~ «$ je repère que j'arrive à mettre les apprenants en échec simplement parce que le scénario choisi n'est pas conforme à leur niveau ». Dans l'apprentissage intentionnel que constituera la formation des formateurs, l'activité constructive sera première, en appui sur les situations de débriefing observées, c'est-à-dire une « analyse réflexive et rétrospective de l'action» (Pastré et al., 2006). Pour la didactique professionnelle « la connaissance se présente sous deux formes indissociablement liées: une forme opératoire et une forme prédicative » (Pastré et al., 2006). Sa forme prédicative est nécessaire au sujet pour établir des liens entre les éléments constitutifs d'une situation (objets, propriétés et relations) et sa forme opératoire s'exprime par la possibilité donnée au sujet de s'adapter à la situation parce qu'il reconnaît ces éléments.

Ainsi la formation devrait permettre aux formateurs de repérer l'incidence des compétences nontechniques dans la situation observée (registre épistémique) et d'accompagner les formés vers une position réflexive leur permettant d'ajuster les compétences non-techniques (registre pragmatique). C'est-à-dire que la conceptualisation consistera pour les formateurs à repérer des classes de situations à partir d'invariants organisateurs et d'indicateurs (Pastré et al., 2006).

Dans le cadre d'une formation de formateurs, l'accompagnement du changement peut donc se situer à trois niveaux : celui de l'élaboration des scénarios et de leurs objectifs, celui de la construction concertée d'un déroulé de débriefing, et celui du déplacement des ressources et des postures sollicitées par les formateurs à partir de leur champ de compétences professionnelles et de celui des sciences humaines développées par l'analyse des débriefings et le travail de construction des compétences techniques et non-techniques.

Pour les formateurs, la simulation, qui vise notamment à construire les compétences non-techniques, ne doit pas être simplement accompagnée d'une modification des supports pédagogiques, c'està-dire passer du cours traditionnel frontal à la mise en situation simulée. Quel que soit le corps professionnel des formateurs (MAR ou IADE), les postures, la place du formateur et celles des formés sont à réin- terroger dans une perspective interactionnelle visant notamment le développement de la réflexivité en situation de débriefing, de manière à engendrer de réelles constructions des réalités professionnelles et les compétences y afférant. «Ce nouveau paradigme de formation transforme [...] le métier de formateur » (Bastiani, Minveille \& Calmettes, 2015). Partant du principe de coprésence de ces acteurs en situation réelle et en situation de supervision et de débriefing, il nous apparaît essentiel que la formation de formateur soit commune. D'autant qu'il ne s'agit pas de contenus techniques mais bien d'un accompagnement qui vise la réflexivité chez les formés.

Cependant, les formateurs doivent associer des compétences techniques médicales (MAR) et paramédicales (IADE) à des compétences en sciences humaines qui ont un caractère commun pour accompagner le développement des formés dans toutes les dimensions de leurs futures activités. Nous avons pu constater que les interventions des superviseurs lors du débriefing ne sont pas nécessairement spécifiques au corps professionnel. Le superviseur IADE par exemple s'adresse indifféremment à un MAR et à un IADE.

C'est avec cette visée que nous construirons le plan de formation de formateurs dans la deuxième phase de notre programme de recherche.

\section{NOTES}

1. Une présentation courte et partielle du dispositif a été réalisée dans le cadre d'un reportage télévisé, voir [https://www.youtube.com/channel/ UCfNs9So_xTYO5WYTpsFX0Aw]

2. Une activité pas nécessairement essentielle qui peut permettre de contenir l'anxiété liée à la situation de crise. 


\section{RÉFÉRENCES}

Anceaux, F., \& Beuscart-Zéphir, M. C. (2002). La consultation préopératoire en anesthésie : gestion de la prise d'informations et rôle des données retenues dans la planification du processus d'anesthésie. Le Travail Humain, 65, 59-88.

Bastiani, B., Minville, V., \& Calmettes, B. (2015). L'expérience du simulateur en formation : le cas d'un bloc opératoire virtuel. Dans M. Saint-Jean, N. Péoc'h $\&$ B. Bastiani (dir.), Accompagner le changement dans le champ de la santé (p. 51-64). Paris, France: De Boeck Estem.

Bastiani, B., Calmettes, B., \& Minville, V. (2016). Le débriefing en question dans la formation par simulation pleine échelle en santé ; le cas des situations critiques en anesthésie-réanimation. Dans M. SaintJean, N. Lafranchise, C. Lepage \& L. Lafortune. Regards croisés sur la rétroaction et le débriefing : accompagner, former et professionnaliser. Montréal, Québec: Presses universitaires du Québec.

Bronckart, J. P. (2009). La notion de compétences est-elle pertinente en éducation. Repéré à [http://www.skolo. org/spip.php?article1124]

Chiniara, G., \& Pellerin, H. (2014). Simulation et gestion d'une situation de crise. Dans O. Fourcade, T. Geeraerts, V. Minville \& K. Samii (dir.), Traité d'anesthésie et de réanimation (4 ${ }^{\mathrm{e}}$ éd., p. 374-385). Paris, France : Lavoisier.

Clot, Y., \& Faïta, D. (2000). Genre et style en analyse du travail : concepts et méthodes. Travailler, 4, 7-42.

Cooper, S., \& Wakelmam, A (1999). Leadership of resuscitation teams: Lighthouse leadership. Resuscitation, 1, 27-45.

Cooper, J. B., \& Gaba, D (2002). No myth: anesthesia is a model for addressing patient safety. Anesthesiology, 97, $1335-1337$

Cooper, J. B., Newbower, R. S., Long, C. D., \& McPeek, B. (2002). Preventable anesthesia mishaps: a study of human factors. Quality \& Safety in Health Care, 11, 277-283.

Croskerry, A. (2009). Universal Model of Diagnostic Reasoning. Academic Medicine, 84(8), 1022-1028.

Dismukes, R. K. P., \& Smith, G. M. (2001). Facilitation and debriefing in aviation training and operations. Ashgate, 3(3), 134-145.

Endsley, M. R., \& Jones, D. G. (2011). Designing for Situation Awareness: An Approach to User Centered Design. Boca Raton, États-Unis : CRC Press.

Fioratou, E., Flin, R., Glavin, R., \& Patey, R. (2010). Beyond monitoring: distributed situation awareness in anaesthesia. British Journal of Anaesthesia, 105(1), 83-90.

Fletcher, G. C. L., McGeorge, P., Flin, R. H., Glavin, R. J., \& Maran, J. (2002). The role of non technical skills in anesthesia: a review of current literature. British Journal of Anaesthesia, 88(3), 418-429.
Gaba, D. M., \& Deanda, A. (1988). A comprehensive anesthesia simulation environnment: re-creating the operating room for research and training. Anesthesiology, 69(3), 387-94.

Gaba, D. M. (1995). Anesthesia simulators. Canadian journal of anaesthesia, 42(10), 952-953.

Gawande, A., Zinner, M., Studdert, D., \& Brennan, T. (2003). Analysis of errors reported by surgeons at thress teaching hospitals. Surgery, 133, 614-21.

Geeraerts, T. (2009). Évaluation du stress induit par la simulation des situations critiques en Anesthésie-Réanimation chez les internes d'Ile de France (Mémoire de Diplôme Inter-Universitaire, universités Paris 5, Paris 6, Paris 11, Paris 12, Paris, France).

Granry, J. C., \& Moll, M. C. (2012). État de l'art en matière de pratiques de simulation dans le domaine de la santé. Rapport de Mission Haute Autorité de Santé. Repéré à http://www.has-sante.fr/portail/upload/docs/application/pdf/2012-01/simulation_en_sante_-_rapport.pdf

Jaffrelot, M., \& Savoldelli, G. (2013). Concevoir un centre de simulation. Dans S. Boet, J. C. Granry \& G. Savoldelli (dir.), La simulation en santé : de la théorie à la pratique (p. 403-410). Paris, France : Springer.

Lienhart, A., Auroy, Y., Pequignot, F., \& Benhamou, D. (2006). Survey of anesthesia-related mortality in France. Anesthesiology, 105(6), 1087-1097.

Naik, V. N., \& Brien, S. E. (2013). La simulation : une façon d'aborder et d'améliorer la sécurité du patient. Canadian Journal of Anesthesia, 60, 192-200.

Neyns, V. (2011). Les modalités du contrôle cognitif en situation dynamique : anticipation et gestion des dérives. Le cas de l'anesthésie (Thèse de doctorat, université Toulouse 2 Le Mirail, Toulouse, France).

Pastré, P. (1999). Travail et compétences : un point de vue de didacticien. Formation Emploi, 67, 109-125.

Pastré, P. (2002). Lanalyse du travail en didactique professionnelle. Revue Française de Pédagogie, 138, 9-17.

Pastré, P. (2005). Apprendre par la simulation: De l'analyse du travail aux apprentissages professionnels. Toulouse, France : Octares.

Pastré, P., Mayen, P., \& Vergnaud, G. (2006). La didactique professionnelle. Revue française de pédagogie, 154, 145-198.

Pastré, P. (2011). Situation d'apprentissage et conceptualisation. Recherches en Éducation, 12, 12-25.

Perrenoud, P. (1997). Construire des compétences dès l'école. Paris, France : ESF.

Phipps, D., Meakin, G. H., Beatty, P. C. W., Nsoedo, C., \& Parker, D. (2008). Human factors in anaesthetic practice: insights from a task analysis. British Journal of Anaesthesia, 100(3), 333-343.

Reader, T., Flin, R., Lauche, K., \& Cuthbertson, B. H. (2006). Non-technical skills in the intensive care unit. British Journal of Anaesthesia, 96(5), 551-9.

Riem, N., Boët, S., \& Bould, M. D. (2012). Do technical skills correlate with non-technical skills in crisis resource management: a simulation study. British Journal of Anaesthesia, 109, 723-728. 
Rogalski, J. (2004). La didactique professionnelle : une alternative aux approches de « cognition située » et « cognitiviste » en psychologie des acquisitions. Activités, 1(2), 103-118.

Samurçay, R., \& Pastré, P. (1995) La conceptualisation des situations de travail dans la formation des compétences. Éducation Permanente, 123, 13-31.

Schaefer, H. G., Helmreich, R. L., \& Scheidegger, D. (1994). Human factors and safety in emergency medicine. Resuscitation, 28(3), 221-5.

Schulz, C. M., Endsley, M. R., \& Kochs, E. F. (2013). Situation awareness in anesthesia: concept and research. Anesthesiology, 118, 729-742.

Sheridan, T. B. (2008). Risk, Human Error, and System Resilience: Fundamental Ideas. Human Factors, 50(3), 418-426.

Tversky, A., \& Kahneman, D. (1974). Judgment under Uncertainty: Heuristics and Biases. Science, New Series, 185(4157), 1124-1131.

Vergnaud, G. (1990). La théorie des champs conceptuels. Recherches en Didactique des Mathématiques, 10(2-3), 133-170.
Verscheure, I. (2015). Construction du référentiel de compétences non techniques en bloc opératoire : étape préliminaire à l'élaboration d'un serious game. Dans M. Saint-Jean, N. Péoc'h \& B. Bastiani (dir.), Accompagner le changement dans le champ de la santé (p. 65-77). Paris, France : De Boeck Estem.

Von Wyl, T., Zuercher, M., Amsler, F., Walter, B., \& Ummenhofer, W. (2009). Technical and non-technical skills can be reliably assessed during paramedic simulation training. Acta Anaesthesiologica Scandinavica, 53, 121-127.

Weinger, M. B., \& Slagle, J. M. (2001). Task and workload analysis of the clinical performance of anesthesia residents with different levels of experience. Anesthesiology, 95, A1145.

Zigmont, J. J., Kappus, L. J., \& Sudikoff, S. N. (2011). The 3D model of debriefing: defusing, discovering and deepening. Seminars in perinatalogy, 35(2), 52-8. 